\title{
GLOBAL STABILITY OF THE ENDEMIC EQUILIBRIUM AND UNIFORM PERSISTENCE IN EPIDEMIC MODELS WITH SUBPOPULATIONS
}

\author{
XIAODONG LIN ${ }^{1}$ and JOSEPH W.-H. SO ${ }^{2}$
}

(Received 10 October 1990)

\begin{abstract}
We consider the epidemic model with subpopulations introduced in Hethcote [5]. It is shown that if the endemic equilibrium exists, then the system is uniformly persistent. Moreover, the endemic equilibrium is globally asymptotically stable under the assumption of small effective contact rates between different subpopulations.
\end{abstract}

\section{Introduction}

The following system of $3 n$ autonomous ordinary differential equations, taken from Appendix A of Jacquez, Simon, Koopman, Sattenspiel and Perry [9], has been widely used in the study of the spread of infectious diseases (cf. Hethcote [5], Sattenspiel and Simon [13], Lajmanovich and Yorke [10], Hethcote [6], Post, DeAngelis and Travis [12], and Hethcote and Thieme [7]). It includes the general SI, SIS, SIR and SIRS models used in mathematical epidemiology and it takes the form

$$
\begin{aligned}
& x_{i}^{\prime}=b_{i}\left(N_{i}-x_{i}\right)-x_{i} \sum_{j} \lambda_{i j} y_{j}+\kappa_{i} z_{i}, \\
& y_{i}^{\prime}=-\left(\gamma_{i}+b_{i}\right) y_{i}+x_{i} \sum_{. j} \lambda_{i j} y_{j}, \\
& z_{i}^{\prime}=-\left(b_{i}+\kappa_{i}\right) z_{i}+\gamma_{i} y_{i},
\end{aligned}
$$

for $i=1, \ldots, n$, where $x_{i}(0), y_{i}(0)$ and $z_{i}(0) \geq 0$. Here $x_{i}$ (resp. $y_{i}$; resp. $z_{i}$ ) denotes the number of susceptible (resp. infected; resp. recovered)

\footnotetext{
${ }^{1}$ Department of Applied Mathematics, University of Waterloo, Ontario, Canada N2L 3G1.

${ }^{2}$ Department of Mathematics, University of Alberta, Edmonton, Alberta, Canada T6G 2G1.

(C) Copyright Australian Mathematical Society 1992, Serial-fee code 0334-2700/92
} 
individuals in the $i$ th subpopulation. $N_{i}$ (resp. $b_{i}$; resp. $\gamma_{i}$; resp. $\kappa_{i}$ ) is the total size (resp. birth and death rate; resp. recovery rate; resp. rate at which recovered individuals loses immunity) for the $i$ th subpopulation. $\lambda_{i j}$ is the effective contact rate between individuals in the $i$ th subpopulation with individuals in the $j$ th subpopulation. All the parameters $N_{i}, b_{i}, \gamma_{i}, \kappa_{i}, \lambda_{i j}$ are assumed to be non-negative.

An outstanding unsolved problem in mathematical epidemiology is to determine if the endemic equilibrium of (1.1), i.e. an equilibrium of the form $\left(x^{*}, y^{*}, z^{*}\right)$ where $x_{i}^{*}, y_{i}^{*}, z_{i}^{*}>0$, is globally stable. In this paper we shall make a contribution to this global stability question by showing that if the effective contact rates between different subpopulations are small, i.e. $\lambda_{i j}$ $(i \neq j)$ is small, then the endemic equilibrium (if it exists) is globally stable.

The rest of the paper is organised as follows. In Section 2, we set up the necessary notations and state some known results concerning the behaviour of solutions of (1.1). We also include new proofs for the existence, uniqueness and local asymptotic stability of the endemic equilibrium. The question of uniform persistence (as introduced in the mathematical population biology literature) will be considered in Section 3. It is shown that (1.1) is uniformly persistent if and only if the endemic equilibrium exists. In Section 4 , the global stability question will be considered. The endemic equilibrium is shown to be globally stable under the assumption of small effective contact rates between different subpopulations.

\section{Some known results with new proofs}

In this section, we recall some known results concerning (1.1) which were proved in [6] and [7]. We also present new proofs for the existence, uniqueness and local asymptotic stability of the endemic equilibrium.

From now on, we shall always make the following assumptions on the parameters:

(H1) $N_{i}>0$ for all $i$,

(H2) $\gamma_{i}>0$ for all $i$,

(H3) $b_{i}+\kappa_{i}>0$ for all $i$, and

(H4) $\lambda_{i j} \geq 0$ for all $i, j$ and $\lambda_{i j}=0$ if and only if $\lambda_{j i}=0$.

We shall only be interested in (1.1) on the positive cone $\mathbb{R}_{+}^{3 n}$. Clearly, $\mathbb{R}_{+}^{3 n}$ is positively invariant under (1.1).

Let $x=\left(x_{1}, \ldots, x_{n}\right), y=\left(y_{1}, \ldots, x_{n}\right), z=\left(z_{1}, \ldots, z_{n}\right)$ and $N=$ $\left(N_{1}, \ldots, N_{n}\right)$. If we set $w=x+y+z$, then by $(1.1) w_{i}^{\prime}=b_{i}\left(N_{i}-w_{i}\right)$ so 
that $w_{i}(t) \rightarrow N_{i}$ as $t \rightarrow \infty$. The set

$$
S=\left\{(x, y, z) \in \mathbb{R}_{+}^{3 n}: x+y+z=N\right\}
$$

is positively invariant under (1.1). Since we are only interested in asymptotic behavior, we can reduce (1.1) to a system of $2 n$ equations

$$
\begin{aligned}
& y_{i}^{\prime}=-\left(b_{i}+\gamma_{i}\right) y_{i}+\left(N_{i}-y_{i}-z_{i}\right) \sum_{j} \lambda_{i j} y_{j}, \\
& z_{i}^{\prime}=-\left(b_{i}+\kappa_{i}\right) z_{i}+\gamma_{i} y_{i} .
\end{aligned}
$$

Due to the reduction from (1.1) to (2.1), we shall only be interested in solutions of (2.1) lying in the set

$$
B=\left\{(y, z) \in \mathbb{R}_{+}^{2 n}: y+z \leq N\right\} .
$$

The origin $E_{0}=(0,0) \in \mathbb{R}^{2 n}$ is an equilibrium for (2.1), called the nodisease equilibrium. Let

and

$$
\begin{gathered}
\Lambda=\left(\begin{array}{ccc}
\lambda_{11} & \ldots & \lambda_{1 n} \\
\vdots & \ddots & \vdots \\
\lambda_{n 1} & \ldots & \lambda_{n n}
\end{array}\right) \\
\tilde{\Lambda}=\left(\begin{array}{ccc}
N_{1} \lambda_{11} & \ldots & N_{1} \lambda_{1 n} \\
\vdots & \ddots & \vdots \\
N_{n} \lambda_{n 1} & \ldots & N_{n} \lambda_{n n}
\end{array}\right),
\end{gathered}
$$

$$
A=\left(\begin{array}{ccc}
-\left(b_{1}+\gamma_{1}\right) & \ldots & 0 \\
& \ddots & \\
0 & \ldots & -\left(b_{n}+\gamma_{n}\right)
\end{array}\right)+\tilde{\Lambda}
$$

It follows from Perron-Frobenius theory that the eigenvalue, $s(A)$, of $A$ with the largest real part is a real number.

THEOREM 2.1 (Hethcote [6]). The set $B$ is positively invariant under (2.1). If $s(A) \leq 0, E_{0}$ is globally asymptotically stable on $B$. If $s(A)>0, E_{0}$ is unstable on $B$.

THEOREM 2.2 (Hethcote and Thieme [7]). Assume $\Lambda$ is irreducible and $s(A)$ $>0$. Then there exists a unique equilibrium $E^{*}=\left(y^{*}, z^{*}\right)$ of $(2.1)$, called the endemic equilibrium, in the interior $\dot{B}$ of $B$. Furthermore, $E^{*}$ (if it exists) is locally asymptotically stable.

We now give a new proof for Theorem 2.2 by means of the following theorem. It is a slight modification of Theorem 2.1 in Smith [14] and can be proved in a similar way. 
THEOREM 2.3. Given a system of ordinary differential equations

$$
u_{i}^{\prime}=F_{i}\left(u_{1}, \ldots, u_{n}\right) \quad(i=1, \ldots, n)
$$

where $F=\left(F_{1}, \ldots, F_{n}\right)$ is $C^{1}$ and $u=\left(u_{1}, \ldots, u_{n}\right) \in \dot{\mathbb{R}}_{+}^{n}$. Assume

(i) $\partial F_{i} / \partial u_{j} \geq 0 \quad(i \neq j)$,

(ii) $u \geq v \geq 0$ implies $D F(v) \geq D F(u)$, where $D F(u)$ denotes the derivative (Jacobian) of $F$ at $u$, and

(iii) given any $\varepsilon>0$, there exists a vector $v \in \mathbb{R}^{n}$ such that $0<v_{i}<\varepsilon$ for all $i$ and $F(v)>0$.

Then (2.5) has at most one positive equilibrium. If there is no positive equilibrium, every solution is unbounded. If there is a positive equilibrium, this equilibrium is globally asymptotically stable over $\dot{\mathbb{R}}_{+}^{n}$.

Proof of Theorem 2.2. We need to solve

$$
-\left(b_{i}+\gamma_{i}\right) y_{i}+\left(N_{i}-y_{i}-z_{i}\right) \sum_{j} \lambda_{i j} y_{j}=0
$$

and

$$
-\left(b_{i}+\kappa_{i}\right) z_{i}+\gamma_{i} y_{i}=0
$$

where $y_{i}, z_{i}>0$ and $y_{i}+z_{i}<N_{i}$ for all $i$. From (2.7), $z_{i}=\frac{\gamma_{i}}{b_{i}+\kappa_{i}} y_{i}$. Substituting this into (2.6), we obtain

$$
\frac{N_{i} \sum_{k} \lambda_{i k} y_{k}}{\left(b_{i}+\gamma_{i}\right)+\left(1+\frac{y_{i}}{b_{i}+\kappa_{i}}\right) \sum_{k} \lambda_{i k} y_{k}}-y_{i}=0 .
$$

Denote the left-hand side of (2.8) by $F_{i}(y)$ and consider the system

$$
y_{i}^{\prime}=F_{i}\left(y_{1}, \ldots, y_{n}\right) \quad(i=1, \ldots, n) .
$$

We now verify hypotheses (i), (ii) and (iii) in Theorem 2.3 for system (2.9). First of all,

$$
\frac{\partial F_{i}}{\partial y_{j}}=\frac{\left(b_{i}+\gamma_{i}\right) N_{i} \lambda_{i j}}{\left[\left(b_{i}+\gamma_{i}\right)+\left(1+\frac{\gamma_{i}}{b_{i}+\kappa_{i}}\right) \sum_{k} \lambda_{i k} y_{k}\right]^{2}}-\delta_{i j},
$$

where $\delta_{i j}$ is the Kronecker delta. Thus (i) is satisfied. Since $\partial F_{i} / \partial y_{j}$ is decreasing with respect to each of its variables $y_{1}, \ldots, y_{n}$, (ii) is also satisfied. As for (iii), since $A$ is an irreducible matrix with non-negative off-diagonal entries, there exists a positive eigenvector $v=\left(v_{1}, \ldots, v_{n}\right)>0$ of $A$ corresponding to the eigenvalue $s(A)$, i.e.

$$
-\left(b_{i}+\gamma_{i}\right) v_{i}+N_{i} \sum_{j} \lambda_{i j} v_{j}=s(A) v_{i} \quad \text { for all } i \text {. }
$$


Hence, for any number $p>0$,

$$
F_{i}(p v)=\frac{p v_{i}\left[s(A) N_{i}-p v_{i}\left(1+\frac{\gamma_{i}}{b_{i}+\kappa_{i}}\right)\left(s(A)+b_{i}+\gamma_{i}\right)\right]}{\left(b_{i}+\gamma_{i}\right) N_{i}+p v_{i}\left(1+\frac{\gamma_{i}}{b_{i}+\kappa_{i}}\right)\left(s(A)+b_{i}+\gamma_{i}\right)} .
$$

Now $s(A)>0$ implies $F_{i}(p v)>0$ for $p$ sufficiently small. Thus (iii) is also satisfied. Finally, since $F_{i}\left(y_{1}, \ldots, y_{n}\right) \leq N_{i}-y_{i},(2.9)$ cannot have unbounded solutions. The existence and uniqueness of the endemic equilibrium, $E^{*}$, for (2.1) now follow immediately from Theorem 2.3 . Of course, one needs to show $y_{i}^{*}+z_{i}^{*}<N_{i}$ for all $i$ but that is clear from (2.6).

To show that $E^{*}$ is (locally) asymptotically stable, we first note that by (2.6)

$$
-\left(b_{i}+\gamma_{i}\right) y_{i}^{*}+\left(N_{i}-y_{i}^{*}-z_{i}^{*}\right) \sum_{j} \lambda_{i j} y_{j}^{*}=0 \quad(i=1, \ldots, n) .
$$

Let

$$
\begin{aligned}
& M=\left(\begin{array}{ccc}
-\left(b_{1}+\gamma_{1}\right) & \ldots & 0 \\
& \ddots & \\
0 & \ldots & -\left(b_{n}+\gamma_{n}\right)
\end{array}\right) \\
& +\left(\begin{array}{ccc}
N_{1}-y_{1}^{*}-z_{1}^{*} & \ldots & 0 \\
& \ddots & \\
0 & \ldots & N_{1}-y_{n}^{*}-z_{n}^{*}
\end{array}\right) \Lambda .
\end{aligned}
$$

Then $M$ is irreducible and has non-negative off-diagonal entries. Moreover, by (2.10), $M y^{*}=0$. Thus $s(M) \leq 0$. Consequently, there exists a diagonal matrix $C=\operatorname{diag}\left\{c_{1}, \ldots, c_{n}\right\}$ with $c_{i}>0$ for all $i$ such that $s\left(C M+M^{\top} C\right)$ $\leq 0$. The Jacobian matrix of right hand side of $(2.1)$ at $E^{*}$ is given by

$$
Q=\left(\begin{array}{cc}
M+J & J \\
\Gamma & K
\end{array}\right)
$$

where $J=\operatorname{diag}\left\{-\sum_{j} \lambda_{1 j} y_{j}^{*}, \ldots,-\sum_{j} \lambda_{n j} y_{j}^{*}\right\}, \Gamma=\operatorname{diag}\left\{\gamma_{1}, \ldots, \gamma_{n}\right\}$ and $K=\operatorname{diag}\left\{-\left(b_{1}+\kappa_{1}\right), \ldots,-\left(b_{n}+\kappa_{n}\right)\right\}$. Define $S=\operatorname{diag}\{C, D\}$, where $D=\operatorname{diag}\left\{d_{1}, \ldots, d_{n}\right\}$ and $d_{i}=\left(c_{i} / \gamma_{i}\right) \sum_{j} \lambda_{i j} y_{j}^{*}>0$. Then

$$
S Q+Q^{\top} S=\left(\begin{array}{cc}
C M+M^{\top} C+2 C J & 0 \\
0 & 2 D K
\end{array}\right) .
$$

Since

$$
\begin{gathered}
C J=\operatorname{diag}\left\{-c_{1} \sum_{j} \lambda_{1 j} y_{j}^{*}, \ldots,-c_{n} \sum_{j} \lambda_{n j} y_{j}^{*}\right\}, \\
D K=\operatorname{diag}\left\{-\frac{c_{1}\left(b_{1}+\kappa_{1}\right)}{\gamma_{1}} \sum_{j} \lambda_{1 j} y_{j}^{*}, \ldots,-\frac{c_{n}\left(b_{n}+\kappa_{n}\right)}{\gamma_{n}} \sum_{j} \lambda_{n j} y_{j}^{*}\right\},
\end{gathered}
$$


and $s\left(C M+M^{\top} C\right) \leq 0$, therefore $S Q+Q^{\top} S$ is a stable matrix. A wellknown theorem of Lyapunov (cf. [11]) shows that $Q$ is a stable matrix. Hence the endemic equilibrium is locally asymptotically stable.

REMARK. In the case when $\Lambda$ is reducible, system (2.1) decouples into two or more (smaller) irreducible subsystems, by (H4). We can then apply Theorem 2.2 to each of these irreducible subsystems. Thus, the endemic equilibrium, if it exists, must be unique and is locally asymptotically stable. Moreover, the endemic equilibrium exists if and only if $s\left(A_{k}\right)>0$ for all $k$, where $A_{k}$ is the $A$ for the $k$ th irreducible subsystem.

Following immediately from Theorem 2.2, we have

THEOREM 2.4. If $\Lambda$ is irreducible and $s(A)>0$, then there is a unique positive equilibrium $\left(x^{*}, y^{*}, z^{*}\right)$ of $(1.1)$ and it is locally asymptotically stable.

REMARK. As in the case for (2.1), the endemic equilibrium for (1.1), if it exists, is asymptotically stable, irrespective of whether $\Lambda$ is irreducible or not.

\section{Uniform persistence}

In the last section it was shown that the endemic equilibrium is locally asymptotically stable when it exists. Hereafter, we shall study the global asymptotic behaviour of solutions of $(2.1)$ in the positively invariant set $B$. In this section, we show that if the endemic equilibrium exists, then the number of each group (susceptible, infected and removed) in each subpopulation will remain above a certain positive level. In other words, each group in each subpopulation persists. If, in addition, $\Lambda$ is irreducible and the disease exists in any subpopulation, then it will spread immediately to all subpopulations.

Let

$$
B_{1}=\left\{\left(0, \ldots, 0, z_{1}, \ldots, z_{n}\right): 0 \leq z_{i} \leq N_{i} \quad(i=1, \ldots, n)\right\} .
$$

Then $B_{1}$ is positively invariant under $(2.1)$ and is negatively invariant relative to $B . B_{1}$ is referred to as the no-disease set.

Our first result says that if the disease exists in any one of the subpopulations, then it will spread immediately to all subpopulations and remains in every subpopulation from then on.

Theorem 3.1. Assume $\Lambda$ is irreducible. Let $(y(t), z(t))$ be a solution of (2.1) in $B$. If $(y(0), z(0)) \in B \backslash B_{1}$, then $(y(t), z(t)) \in \dot{B}$ for all $t>0$, where $\dot{B}$ denotes the interior of $B$. 
Before we prove this theorem, we need the following two lemmas whose proofs are standard.

Lemma 3.2. If $(y(0), z(0)) \in \dot{B}$, then $(y(t), z(t)) \in \dot{B}$ for all $t \geq 0$.

LemmA 3.3. If $(y(0), z(0)) \in \partial B \backslash B_{1}$, then there exists $\delta>0$ such that

$$
(y(t), z(t)) \in \dot{B} \quad \text { for all } 0<t<\delta .
$$

Proof of Theorem 3.1. Let $(y(0), z(0)) \in B \backslash B_{1}$. If $(y(0), z(0)) \in \dot{B}$, then $(y(t), z(t)) \in \dot{B}$ for $t \geq 0$ by Lemma 3.2. If $(y(0), z(0)) \in \partial B \backslash B_{1}$, by Lemma 3.3 there exists $\delta>0$ such that $(y(t), z(t)) \in \dot{B}$ for all $0<t<\delta$. Hence $(y(t), z(t)) \in \dot{B}$ for all $t>0$.

It is known that if $s(A) \leq 0$ then the no-disease equilibrium $E_{0}$ is the unique equilibrium and it is globally asymptotically stable on $B$. When $s(A)>0$, we have the following result.

Theorem 3.4. If $\Lambda$ is irreducible and $s(A)>0$, then (2.1) is uniformly persistent in $B$ with respect to $\partial B$. That is, there is an $\eta>0$ such that $\liminf _{t \rightarrow \infty} y_{i}(t) \geq \eta, \liminf _{t \rightarrow \infty} z_{i}(t) \geq \eta$, and $\operatorname{lim\operatorname {sup}_{t\rightarrow \infty }} y_{i}(t)+z_{i}(t) \leq$ $N_{i}-\eta$, for all solution $(y(t), z(t))$ with initial condition in $B \backslash B_{1}$.

The biological interpretation of Theorem 3.4 is that if the threshold, $s(A)$, exceeds zero, the disease will not only exist in every subpopulation but in fact the number of individuals in each group (susceptible, infectious and removed) will always remain beyond a certain positive level $\eta$.

The proof of Theorem 3.4 depends on a theorem in Hofbauer and So [8] which we state below for the sake of easy reference. (See also Butler and Waltman [1] and Garay [4].)

Let $\mathscr{Z}$ be a metric space with metric $d, f: \mathscr{Z} \rightarrow \mathscr{Z}$ be continuous and $\mathscr{Y} \subset \mathscr{Z}$ be closed with $f(\mathscr{X} \backslash \mathscr{Y}) \subset \mathscr{X} \backslash \mathscr{Y}$. Suppose $\mathscr{Z}$ has a compact global attractor $X$ and let $M$ be the maximal compact invariant set in $\mathscr{Y}$. Then we have

THEOREM 3.5 (Hofbauer and So [8]). $f$ is uniformly persistent with respect to $\mathscr{Y}$ if and only if

1. $M$ is isolated in $X$, and

2. $W^{s}(M) \subset \mathscr{Y}$, where $W^{s}(M)$ denotes the stable set of $M$.

Proof of Theorem 3.4. Let $\mathscr{X}=B, \mathscr{Y}=\partial B$ and $f$ be the time one map of the flow defined by (2.1). It follows from Theorem 3.1 that $f(\mathscr{X} \backslash \mathscr{Y}) \subset$ 
$\mathscr{Z} \backslash \mathscr{Y}$. Clearly $X=\omega(B)$, where $\omega(B)$ is the $\omega$-limit set of $B$, a global attractor of $\mathscr{Z}$. Let $M$ be the maximal compact invariant set in $\mathscr{Y}$.

Claim. $M=\left\{E_{0}\right\}$.

Suppose not, then there exists $\left(y^{0}, z^{0}\right) \in M$ and either (i) $\left(y^{0}, z^{0}\right) \in$ $B \backslash B_{1}$ or (ii) $\left(y^{0}, z^{0}\right) \in B_{1} \backslash\left\{E_{0}\right\}$. Let $(y(t), z(t))$ be the solution with initial condition $\left(y^{0}, z^{0}\right)$. If (i) holds then $(y(t), z(t)) \in \dot{B}$, by Theorem 3.1, contradicting $M \subset \partial B$. On the other hand, if (ii) holds then the solution must take the form

$$
(y(t), z(t))=\left(0, \ldots, 0, c_{1} e^{-\left(b_{1}+\kappa_{1}\right) t}, \ldots, c_{1} e^{-\left(b_{n}+\kappa_{n}\right) t}\right)
$$

where $c_{1}, \ldots, c_{n}$ are not all zero. Clearly for $t$ sufficiently negative, $(y(t)$, $z(t)) \notin B$, which contradicts the invariance of $M$.

In order to show uniform persistence, it suffices to verify conditions 1 and 2 in Theorem 3.5. We will do this by constructing a suitable Lyapunov function. Let $V(y)=v^{\top} y$ where $v=\left(v_{1}, \ldots, v_{n}\right)$ is a positive eigenvector of $A^{\top}$ corresponding to the eigenvalue $s(A)$. Then there exists $a>0$ such that $V(y) \geq a\|y\|$ for all $y \geq 0$, where $\|y\|=\max _{i}\left\{\left|y_{i}\right|\right\}$. Since $s(A)>0$ and the derivative of $V$ along solutions is

$$
V^{\prime}=s(A) v^{\top} y-\sum_{i} v_{i}\left(y_{i}+z_{i}\right) \sum_{j} \lambda_{i j} y_{j},
$$

$V^{\prime}>0$ in a neighbourhood $N$ of $E_{0}$ relative to $B \backslash B_{1}$. It follows that any solution in $N$ must leave $N$ at a finite time. Consequently, $M$ is isolated and the stable set of $M, W^{s}(M)$, is equal to $B_{1}$.

REMARK. In the case when $\Lambda$ is reducible, by using Theorem 3.4 and following the same line of reasoning as in the Remark following Theorem 2.2, one can easily show that if the endemic equilibrium exists, then (2.1) is uniformly persistent with respect to $\partial B$.

THEOREM 3.6. If the endemic equilibrium $\left(x^{*}, y^{*}, z^{*}\right)$ exists, then (1.1) is uniformly persistent, i.e. there exists $\eta>0$ such that for all $i$ we have, $\liminf _{t \rightarrow \infty} x_{i}(t) \geq \eta, \liminf _{t \rightarrow \infty} y_{i}(t) \geq \eta$, and $\liminf _{t \rightarrow \infty} z_{i}(t) \geq \eta$, for all solutions $(x(t), y(t), z(t))$ of $(1.1)$ with $(x(0), y(0), z(0)) \in \mathbb{R}_{+}^{3 n} \backslash S_{0}$, where

$$
S_{0}=\left\{(x, y, z) \in \mathbb{R}_{+}^{3 n}: y=0\right\} .
$$




\section{Global stability of the endemic equilibrium}

In this section we show that if the effective contact rates between different subpopulations are sufficiently small, i.e. $\lambda_{i j}(i \neq j)$ is small, then the endemic equilibrium $E^{*}=\left(y^{*}, z^{*}\right)$ for (2.1) (if it exists) must be globally stable. Our approach is to construct a Lyapunov function $V(y, z)$ for the case when $\lambda_{i j}=0$ for all $i \neq j$ and show that this function $V$ continues to be a Lyapunov function when $\lambda_{i j}(i \neq j)$ is small but not neccessarily identical to zero.

Let $U_{i}=y_{i}-y_{i}^{*}-y_{i}^{*} \ln \left(y_{i} / y_{i}^{*}\right)$ and $W_{i}=\left(z_{i}-z_{i}^{*}\right)^{2}$. The functions $U_{i}$ have been used by many authors (cf. So [15] and Freedman and So [3]). Since $x_{i}=N_{i}-y_{i}-z_{i}, x_{i}^{*}=N_{i}-y_{i}^{*}-z_{i}^{*},-\left(y_{i}+b_{i}\right)+x_{i}^{*} \lambda_{i i}+\left(x_{i}^{*} / y_{i}^{*}\right) \sum_{j \neq i} \lambda_{i j} y_{j}^{*}=0$ and $-\left(b_{i}+\kappa_{i}\right) z_{i}^{*}+\gamma_{i} y_{i}^{*}=0$, we have (after some simplications)

$$
U_{i}^{\prime}=\left(y_{i}-y_{i}^{*}\right)\left[-\lambda_{i i}\left(y_{i}-y_{i}^{*}\right)-\lambda_{i i}\left(z_{i}-z_{i}^{*}\right)+\frac{x_{i}}{y_{i}} \sum_{j \neq i} \lambda_{i j} y_{j}-\frac{x_{i}^{*}}{y_{i}^{*}} \sum_{j \neq i} \lambda_{i j} y_{j}^{*}\right]
$$

and

$$
W_{i}^{\prime}=2\left(z_{i}-z_{i}^{*}\right)\left[-\left(b_{i}+\kappa_{i}\right)\left(z_{i}-z_{i}^{*}\right)+\gamma_{i}\left(y_{i}-y_{i}^{*}\right)\right] \text {. }
$$

Define $V=\sum_{i} c_{i} U_{i}+\sum_{i} W_{i}$, where $c_{i}=2 \gamma_{i} / \lambda_{i i}$ (assuming $\lambda_{i i}>0$ for all $i)$. Then $V \geq 0$ and $V=0$ if and only if $(y, z)=\left(y^{*}, z^{*}\right)$. Moreover, (after some simplications)

$$
\begin{aligned}
V^{\prime}= & -\sum_{i} 2 \gamma_{i} \lambda_{i i}\left(y_{i}-y_{i}^{*}\right)^{2}-2 \sum_{i}\left(b_{i}+\kappa_{i}\right)\left(z_{i}-z_{i}^{*}\right)^{2} \\
& +\sum_{i} \frac{2 \gamma_{i}}{\lambda_{i i}}\left(y_{i}-y_{i}^{*}\right)\left[\frac{x_{i}}{y_{i}} \sum_{j \neq i} \lambda_{i j} y_{j}-\frac{x_{i}^{*}}{y_{i}^{*}} \sum_{j \neq i} \lambda_{i j} y_{j}^{*}\right] .
\end{aligned}
$$

Case 1. $\lambda_{i j}=0$ for all $i \neq j$. System (2.1) decouples into $n$ subsystems. The $i$ th subsystem involves only $y_{i}$ and $z_{i} . E^{*}$ exists if and only if

$$
\lambda_{i i} N_{i}>\gamma_{i}+b_{i} \text { for all } i \text {. }
$$

In that case,

From (4.1),

$$
y_{i}^{*}=\frac{\lambda_{i i} N_{i}-\left(\gamma_{i}+b_{i}\right)\left(b_{i}+\kappa_{i}\right)}{b_{i}+\gamma_{i}+\kappa_{i}}, \quad z_{i}^{*}=\frac{\lambda_{i i} N_{i}-\left(\gamma_{i}+b_{i}\right) \gamma_{i}}{b_{i}+\gamma_{i}+\kappa_{i}} .
$$

$$
V^{\prime}=-2 \sum_{i} \gamma_{i} \lambda_{i i}\left(y_{i}-y_{i}^{*}\right)^{2}-2 \sum_{i}\left(b_{i}+\kappa_{i}\right)\left(z_{i}-z_{i}^{*}\right)^{2}
$$

so that $V^{\prime} \leq 0$ and $V^{\prime}=0$ if and only if $(y, z)=\left(y^{*}, z^{*}\right)$. Hence, $V$ is a Lyapunov function for (2.1) and $E^{*}$ is globally stable over $\dot{B}$.

Case 2. $\lambda_{i j}(i \neq j)$ is small. We regard $\lambda_{i i}>0(i=1, \ldots, n)$ as fixed. 
THEOREM 4.1. Assume (4.2) holds. Then provided $\lambda_{i j} \geq 0 \quad(i \neq j)$ are sufficiently small and $(\mathrm{H} 4)$ is satisfied, the endemic equilibrium $E^{*}$ is globally stable over $\dot{B}$.

Before we present the proof of Theorem 4.1, we need the following proposition whose proof was inspired by an argument in Garay [4] as well as a discussion with Josef Hofbauer of University of Vienna and James Selgrade of North Carolina State University.

Proposition 4.2. Let $\pi_{\mu}: \mathbb{R}_{+} \times X \rightarrow X$ be a family of continuous semiflow on a compact metric space $X$ with metric $d$, where $\mu \in P$ and $P$ is a metric space with metric $d_{P}$. Assume $\pi: P \times \mathbb{R}_{+} \times X \rightarrow X$ defined by $\pi(\mu, t, x)=$ $\pi_{\mu}(t, x)$ is continuous. Let $\left\{\mu_{i}\right\}$ be a sequence in $P$ converging to some $\mu_{0} \in P$, as $i$ tends to $\infty$. If corresponding to each $\mu_{i}(i=1,2, \ldots)$, there is a compact subset $\mathscr{A}_{i}$ of $X$ which is invariant and chain-transitive under $\pi_{\mu_{i}}$ and $\mathscr{A}_{i}$ converges to some subset $\mathscr{A}$ of $X$ under the Hausdorff metric $d_{H}$ on $X$ as $i$ tends to $\infty$, then the limiting set $\mathscr{A}$ is compact, invariant and chain-transitive under $\pi_{\mu_{0}}$.

Proof. The verification of the compactness and $\pi_{\mu_{0}}$-invariance of $\mathscr{A}$ is straightforward. To show that $\mathscr{A}$ is chain-transitive under $\pi_{\mu_{0}}$, let $y, z \in$ $\mathscr{A}$ and let $\varepsilon, T>0$ be given. We need to show there is a $(\varepsilon, T)$ chain from $x$ to $y$. Since $\pi$ is continuous and $X$ is compact, there exists $0<$ $\delta<\varepsilon / 3$ such that $d\left(\pi_{\mu_{i}}\left(x_{1}, t\right), \pi_{\mu_{0}}\left(x_{2}, t\right)\right) \leq \varepsilon / 3$ whenever $d\left(x_{1}, x_{2}\right)<\delta$, $d_{P}\left(\mu_{i}, \mu_{0}\right)<\delta$ and $t \in[0,2 T]$. Let $i$ be such that $d_{P}\left(\mu_{i}, \mu_{0}\right)<\delta$ and $d_{H}\left(\mathscr{A}_{i}, \mathscr{A}\right)<\delta$. Then there exist $p, q \in \mathscr{A}_{i}$ such that $d(p, y)<\delta$ and $d(q, z)<\delta$. Since $\mathscr{A}_{i}$ is chain transitive under $\pi_{\mu_{i}}$, there is a $(\varepsilon / 3, T)$ chain from $p$ to $q$. That is, there exist $p_{0}, \ldots, p_{n+1} \in \mathscr{A}_{i}$ with $p_{0}=p$ and $p_{n+1}=q$ and $t_{0}, \ldots, t_{n} \geq T$ such that $d\left(\pi_{\mu_{i}}\left(t_{j}, p_{j}\right), p_{j+1}\right)<\varepsilon / 3$ for all $j=1, \ldots, n$. Without loss, we can assume $t_{j} \leq 2 T$ for all $j$. Since $d_{H}\left(\mathscr{A}_{i}, \mathscr{A}\right)<\delta$, there exist $y_{0}, \ldots, y_{n+1} \in \mathscr{A}$ with $y_{0}=y$ and $y_{n+1}=z$ such that $d\left(y_{j}, p_{j}\right)<\delta$ for all $j=1, \ldots, n+1$. Now,

$$
\begin{aligned}
d\left(\pi_{\mu_{0}}\left(y_{j}\right), y_{j+1}\right) & \leq d\left(\pi_{\mu_{0}}\left(y_{j}\right), \pi_{\mu_{i}}\left(p_{j}\right)\right)+d\left(\pi_{\mu_{i}}\left(p_{j}\right), p_{j+1}\right)+d\left(p_{j+1}, y_{j+1}\right) \\
& \leq \frac{\varepsilon}{3}+\frac{\varepsilon}{3}+\delta<\varepsilon
\end{aligned}
$$

so that $y_{0}, \ldots, y_{n+1}$ and $t_{0}, \ldots, t_{n}$ is a $(\varepsilon, T)$ chain in $A$ from $y$ to $z$. Proof of Theorem 4.1. From (4.2) and Theorem 2.2, we know that $E^{*}$ exists and is asymptotically stable, provided $\lambda_{i j}=0$ for all $i \neq j$. By 
implicit function theorem, $E^{*}$ continues to exist for sufficiently small $\lambda_{i j} \geq 0$ $(i \neq j)$.

According to (4.1), after some simplifications,

$$
\begin{aligned}
V^{\prime}= & -\sum_{i}\left[2 \gamma_{i}+\frac{2 \gamma_{i}}{\lambda_{i i}} \frac{\sum_{j \neq i} \lambda_{i j} y_{j}}{y_{i}}+\frac{2 \gamma_{i}}{\lambda_{i i}} \frac{x_{i}^{*}}{y_{i}^{*}} \frac{\sum_{j \neq i} \lambda_{i j} y_{j}}{y_{i}}\right]\left(y_{i}-y_{i}^{*}\right)^{2} \\
& -2 \sum_{i}\left(b_{i}+\kappa_{i}\right)\left(z_{i}-z_{i}^{*}\right)^{2}-\sum_{i} \frac{2 \gamma_{i}}{\lambda_{i i}} \frac{\sum_{j \neq i} \lambda_{i j} y_{j}}{y_{i}}\left(y_{i}-y_{i}^{*}\right)\left(z_{i}-z_{i}^{*}\right) \\
& +\sum_{i} \sum_{j \neq i} \frac{2 \gamma_{i}}{\lambda_{i i}} \frac{x_{i}^{*}}{y_{i}^{*}} \lambda_{i j}\left(y_{i}-y_{i}^{*}\right)\left(y_{j}-y_{j}^{*}\right)
\end{aligned}
$$

Therefore, $V^{\prime} \leq 0$ and $V^{\prime}=0$ if and only if $(y, z)=\left(y^{*}, z^{*}\right)$, provided the associated quadratic form involving $\left(y_{1}-y_{1}^{*}\right), \ldots,\left(y_{n}-y_{n}^{*}\right),\left(z_{1}-z_{1}^{*}\right), \ldots$ and $\left(z_{n}-z_{n}^{*}\right)$ is negatively definite.

Claim. There exists $\eta>0$ such that $\liminf _{t \rightarrow \infty} y_{i}(t)>\eta$ for all $i$, and for all solutions $(y(t), z(t))$ of (2.1) with $(y(0), z(0)) \in \dot{\mathbb{R}}_{+}^{2 n}$ where $\lambda_{i j} \geq 0$ $(i \neq j)$ are sufficiently small.

Suppose not, then there exists a sequence of parameters $\left\{\mu^{k}\right\}_{k=1}^{\infty}$ where $\mu^{k}=\left(\lambda_{i j}^{k}\right)_{i \neq j}$ and a sequence of solutions $\left\{\left(y^{k}(t), z^{k}(t)\right)\right\}_{k=1}^{\infty}$ of $(2.1)$ corresponding to $\mu^{k}$ with $\left(y^{k}(0), z^{k}(0)\right) \in \dot{B}$ such that $\liminf _{t \rightarrow \infty} y_{j}^{k}(t) \rightarrow 0$ as $k \rightarrow \infty$, for some $i$. Let $\mathscr{A}_{k}=\omega\left(\left(y^{k}(0), z^{k}(0)\right)\right)$, the $\omega$-limit set of the point $\left(y^{k}(0), z^{k}(0)\right)$ under (2.1) corresponding to $\mu^{k}$. By Theorem 3.4, $\mathscr{A}_{k} \subset \dot{B}$ for all $k \geq 1$. By going to a subsequence if necessary, we can assume $\mathscr{A}_{k}$ converges to some subset $\mathscr{A}$ of $X$ under the Hausdroff metric as $k \rightarrow \infty$. By Proposition 4.2, $\mathscr{A}$ is a compact subset of $B$ and is invariant and chain-transitive under (2.1) with $\lambda_{i j}=0$ for all $i \neq j$. We now show that $\mathscr{A}=\left\{E_{0}\right\}$. Since $d\left(\mathscr{A}_{k}, \partial B\right) \rightarrow 0, d(\mathscr{A}, \partial B)=0$. On the other hand, since $E^{*}$ is globally stable over $\dot{B}$ for the case $\lambda_{i j}=0 \quad(i \neq j)$ and $\mathscr{A}$, being chain-transitive, cannot contain a non-trivial attractor, therefore $\mathscr{A}$ cannot contain a point in $\dot{B}$. Thus $\mathscr{A} \subset \partial B$. Similarly, $\mathscr{A}$ cannot contain any point in $\partial B$ except $E_{0}$. Hence $\mathscr{A}=\left\{E_{0}\right\}$ and $\mathscr{A}_{k}$ converges to $E_{0}$ as $k \rightarrow \infty$. However, if $y_{i}, z_{i}<\varepsilon / 2$, by (2.1) we have

$$
\begin{aligned}
y_{i}^{\prime} & =-\left(b_{i}+\kappa_{i}\right) y_{i}+\left(N_{i}-y_{i}-z_{i}\right) \sum_{j} \lambda_{i j} y_{j} \\
& \geq-\left(b_{i}+\kappa_{i}\right) y_{i}+\left(N_{i}-\varepsilon\right) \sum_{j} \lambda_{i j} y_{j} \\
& \geq\left(N_{i} \lambda_{i i}-b_{i}-\kappa_{i}-\varepsilon \lambda_{i j}\right) y_{i} .
\end{aligned}
$$


By assumption (4.2), $N_{i} \lambda_{i i}-b_{i}-\kappa_{i}-\varepsilon \lambda_{i i}$ is positive for sufficiently small $\varepsilon$ and thus $y_{i}^{\prime}>0$. Since $\mathscr{A}_{k} \subset \dot{B}$, this shows that it is impossible to have $\mathscr{A}_{k}$ converging to $E_{0}$ as $k \rightarrow \infty$. This contradiction establishes the claim.

By the above claim, we can assume (without loss of generality) that the coefficient in the third summation of (4.3), i.e.

$$
-\frac{2 \gamma_{i}}{\lambda_{i i}} \frac{\sum_{j \neq i} \lambda_{i j} y_{j}}{y_{i}}
$$

is bounded. Moreover, it tends to 0 as $\lambda_{i j} \rightarrow 0 \quad(i \neq j)$. On the other hand, it is clear that the coefficient in the fourth summation of (4.3), i.e. $\left(2 \gamma_{i} / \lambda_{i i}\right)\left(x_{i}^{*} / y_{i}^{*}\right) \lambda_{i j}$, also tends to 0 as $\lambda_{i j} \rightarrow 0 \quad(i \neq j)$. Thus, provided $\lambda_{i j}$ $(i \neq j)$ is sufficiently small, the associated quadratic form associated with (4.3) is negatively definite and thus $V$ is a Lyapunov function.

Remark. Theorem 4.1 is a perturbation result, and as such is not surprising. However, since a global conclusion is asserted, it does not follow directly from the usual perturbation theorems. As an example, we consider

$$
x^{\prime}=f(x):=x^{2}(1-x)
$$

and

$$
x^{\prime}=g_{\varepsilon}(x):=x(x-\varepsilon)(1-x) .
$$

Then the equilibrium $x=1$ is globally stable (over $\dot{\mathbb{R}}_{+}$) for $f$ but not globally stable for $g_{\varepsilon}$. Moreover, $g_{\varepsilon}$ is $C^{0}$-close to $f$ on any compact interval.

THEOREM 4.3. If the endemic equilibrium $E^{*}$ for (2.1) is globally stable over $\dot{B}$, then the endemic equilibrium $\left(x^{*}, y^{*}, z^{*}\right)$ for (1.1) is globally stable over $\dot{\mathbb{R}}_{+}^{3 n}$.

Proof. Let us first assume $\Lambda$ is irreducible. Given any solution $(x(t), y(t)$, $z(t))$ of $(1.1)$ with $(x(0), y(0), z(0)) \in \dot{\mathbb{R}}_{+}^{3 n}$, its $\omega$-limit set $\Omega$ must be contained in $S$, since $x(t)+y(t)+z(t) \rightarrow N$ as $t \rightarrow \infty$. We will show that $\Omega=\left\{\left(x^{*}, y^{*}, z^{*}\right)\right\}$.

Claim. $\Omega \neq\{(N, 0,0)\}$. Suppose not, then $x(t) \rightarrow N$ and $y(t) \rightarrow 0$ as $t \rightarrow \infty$. Let $V(y)=v^{\top} y$ be the Lyapunov function used in the proof of Theorem 2.4. Then

$$
V^{\prime}=s(A) v^{\top} y-\sum_{i} v_{i}\left(N_{i}-x_{i}\right) \sum_{j} \lambda_{i j} y_{j}
$$


Since $y(t) \geq 0$ and $y(t) \neq 0$ for all $t>0$, then $V^{\prime}>0$ for sufficiently large $t$. This contradicts $y(t) \rightarrow 0$ as $t \rightarrow \infty$.

Hence, $\Omega$ must contain a point $\left(x^{0}, y^{0}, z^{0}\right) \in S$ with either (i) $y^{0}=0$ or (ii) $y^{0}>0$. If (i) holds, since $\Omega$ is invariant, it contains the negative orbit through $\left(x^{0}, y^{0}, z^{0}\right)$. However, this orbit is unbounded and this contradicts the compactness of $\Omega$. Thus (i) is impossible. On the other hand, if (ii) holds, since $(N, 0,0)$ is globally stable over $S \backslash S_{1}$, where

$$
S_{1}=\left\{(x, y, z) \in \mathbb{R}_{+}^{3 n}: y=0\right\},
$$

then $\left(x^{*}, y^{*}, z^{*}\right) \in \Omega$. However, since $\Omega$ is chain-transitive and $\left(x^{*}, y^{*}, z^{*}\right)$ is asymptotically stable, we have $\Omega=\left\{\left(x^{*}, y^{*}, z^{*}\right)\right\}$, as desired.

In the case when $\Lambda$ is reducible, all we need is to apply the above argument to each of the irreducible subsystems as was discussed in the remark following the proof of Theorem 2.2.

RemarK. Assume (4.2) holds. Then provided $\lambda_{i j} \geq 0(i \neq j)$ are sufficiently small and (H4) is satisfied, the endemic equilibrium $\left(x^{*}, y^{*}, z^{*}\right)$ for (1.1) is globally stable over $\mathbb{R}_{+}^{3 n} \backslash S_{0}$.

\section{Acknowledgement}

Research partially supported by Natural Sciences and Engineering Research Council of Canada, grant number NSERC OGP36475.

\section{References}

[1] G. J. Butler and P. Waltman, "Persistence in dynamical systems", J. Diff. Eqns. 63 (1986) 255-263.

[2] M. Fiedler and V. Pták, "On matrices with non-positive off-diagonal elements and positive principle minors", Czechoslovak Math. J. 12 (1962) 382-400.

[3] H. I. Freedman and J. W.-H. So, "Global stability and persistence of simple food chains", Math. Biosci. 76 (1985) 69-86.

[4] B. M. Garay, "Uniform persistence and chain recurrence", J. Math. Anal. Appl. 139 (1989) 372-381.

[5] H. Hethcote, "Qualitative analysis of communicable disease models", Math. Biosci. 28 (1976) 335-356.

[6] H. Hethcote, "An immunization model for a heterogeneous population", Theo. Pop. Biol. 14 (1978) 338-349.

[7] H. Hethcote and H. R. Thieme, "Stability of the endemic equilibrium in epidemic models with subpopulations", Math. Biosci. 75 (1985) 205-277.

[8] J. Hofbauer and J. W.-H. So, "Uniform persistence and repellors for maps", Proc. Amer. Math. Soc. (to appear). 
[9] J. A. Jacquez, C. P. Simon, J. Koopman, L. Sattenspiel and T. Perry, "Modeling and analyzing HIV transmission: the effect of contact patterns", Math. Biosci. 92 (1988) 119-199.

[10] A. Lajmanovich and J. A. Yorke, "A deterministic model for gonorrhea in a nonhomogeneous population", Math. Biosci. 28 (1976) 221-236.

[11] P. Lancaster and M. Tismenetsky, The Theory of Matrices with Applications (2nd ed.), Academic Press, Inc., Orlando (1985).

[12] W. M. Post, D. L. DeAngelis and C. C. Travis, "Endemic disease in environments with spatially heterogeneous host populations", Math. Biosci. 63 (1983) 289-302.

[13] L. Sattenspiel and C. P. Simon, "The spread and persistence of infectious diseases in structured populations", Math. Biosci. 90 (1988) 341-366.

[14] H. L. Smith, "On the asymptotic behavior of a class of deterministic models of cooperating species", SIAM J. Appl. Math. 46 (1986) 368-375.

[15] J. W.-H. So, "A note on the global stability and bifurcation phenomenon of a LotkaVolterra food chain", J. Theo. Bio. 80 (1979) 185-187. 